\title{
Transcription Elongation Factor SPT5
}

National Cancer Institute

\section{Source}

National Cancer Institute. Transcription Elongation Factor SPT5. NCI Thesaurus. Code C17537.

Transcription elong ation factor SPT 5 (1087 aa, 121 kDa) is encoded by the human SUPT $5 \mathrm{H}$ gene. This protein plays a role in both transcriptional regulation and mRNA processing. 